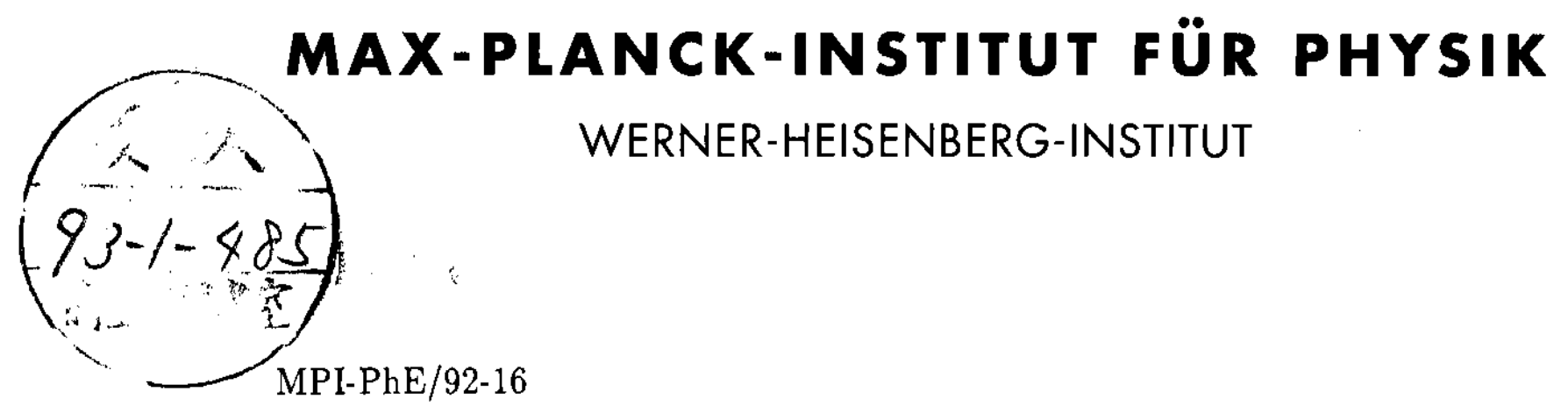

Oktober 1992

\title{
MULTIPLICITY DISTRIBUTIONS IN SMALL PHASE-SPACE DOMAINS IN CENTRAL NUCLEUS-NUCLEUS COLLISIONS
}

\author{
NA35 COLLABORATION
}

\begin{abstract}
J.Bächler ${ }^{8}$.J.Bartke ${ }^{5}$.H.Bialkowska ${ }^{12}$, R.Bock ${ }^{6}, \quad$ R.Brockmann ${ }^{6} . P . B u n c i c^{13}$, S.I.Chase ${ }^{3}$.I.Derado ${ }^{10}$.V.Eckardt ${ }^{10}{ }^{1}$,J.Eschke ${ }^{7}$, D.Ferenc ${ }^{13}, B$. Fleischmann $^{6,7}$, M.Fuchs ${ }^{6,7}$, M.Gaździcki ${ }^{11}$, H.J.Gebauer ${ }^{10}$, E.Gladysz ${ }^{5}$.J.W.Harris ${ }^{3}$. W.Heck ${ }^{7}$, M.Hoffmann ${ }^{3}$.S.Kabana ${ }^{\top}$.K.Kadija ${ }^{13}$, R.Keide ${ }^{9}$, J.Kosiec ${ }^{11}$. M.Kowalski $i^{5}$. A.Kühmichel ${ }^{7}$.M.Lahanas' ${ }^{7}$ Y.Lee ${ }^{7}$.M.Le Vine ${ }^{7},{ }^{14}$..A.Ljubicic jr. ${ }^{13}$.S.Margetis ${ }^{7}$. E.Nappi ${ }^{2}$, G.Odyniec ${ }^{3}$.G.Paic ${ }^{6,13}$. A.D.Panagiotou ${ }^{1,4}$, A.Petridis ${ }^{1}$.J.Pfenning ${ }^{4}$, A.Piper ${ }^{3}$.F.Posa ${ }^{2}$.H.G.Pugh ${ }^{3}$,F.Pühlhofer ${ }^{9}$, G.Rai ${ }^{3}$, W.Rauch ${ }^{10}$.R.Renfordt ${ }^{7}$. D.Röhrich ${ }^{7}$.H.Rothard ${ }^{7} . K$. Runge $^{3}$, A.Sandoval ${ }^{6}$, E.Schmidt ${ }^{7}$, N.Schmitz ${ }^{10}$. E.Schmoet ten ${ }^{8}$,I.Schneider ${ }^{7}$, P.Seyboth ${ }^{10}$, J.Seyerlein $^{10}, \quad$ E.Skrzypczak ${ }^{11}$, P.Stefanski ${ }^{5}$, R.Stock ${ }^{7}$, H.Ströbele ${ }^{7}$, L.Teitelbaum ${ }^{3}, \quad$ S. Tonse ${ }^{3}$, G.Vassileiadis $^{1}$,

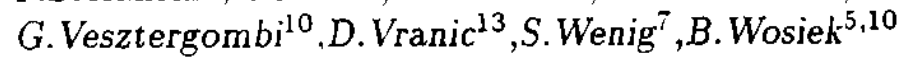

1)Physics Dept.,Univ.of Athens 2)INFN.Sez.Bari and Dipartimento di Fisica. Univ. di Bari 3)Lawrence Berkeley Lab. 4)CERN 5)Inst.of Nuclear Physics.Cracow 6)GSI Darmstadt 7)Fachbereich Physik. Univ.Franfurt 8)Fakultät für Physik. Univ. Freiburg 9)Fachbereich Physik,Univ.Marburg 10)Max-Planck-Institut für Physik, München 11)Inst.of Experimental Physics.Univ. of Warsaw 12)Institute of Nuclear Studies, Warsaw 13)Rudjer Boskovic Inst..Zagreb 
Alle Rechte vorbehalten

Max-Planck-Institut für Physik, München. 


\title{
MULTIPLICITY DISTRIBUTIONS IN SMALL PHASE-SPACE DOMAINS IN CENTRAL NUCLEUS-NUCLEUS COLLISIONS
}

\author{
NA35 COLLABORATION
}

\begin{abstract}
J.Bächler ${ }^{8}, J . B a r t k e^{5}$, H.Bialkowska ${ }^{12}$, R.Bock ${ }^{6}, \quad$ R.Brockmann ${ }^{6}, P$. Buncic $^{13}$, S.I.Chase ${ }^{3}$, I.Derado ${ }^{10}$, V.Eckardt ${ }^{10}$, J.Eschke $^{7}, \quad$ D.Ferenc ${ }^{13}$, B.Fleischmann ${ }^{6,7}$, M.Fuchs ${ }^{6,7}$, M.Gaździcki ${ }^{11}$, H.J.Gebauer ${ }^{10}$, E.Gladysz $^{5}$,J.W.Harris ${ }^{3}$, W.Heck ${ }^{7}$,

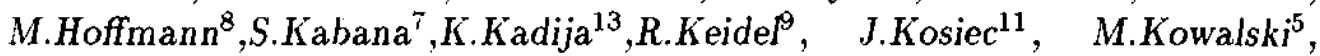
A.Kühmichel ${ }^{7}$, M.Lahanas ${ }^{7}, Y$. Lee $^{7}$, M.Le Vine ${ }^{7},{ }^{14}$, A.L Lubicic jr. ${ }^{13}$, S.Margetis $^{7}$,

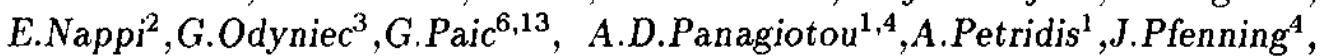
A.Piper ${ }^{9}$, F.Posa ${ }^{2}$,H.G.Pugh ${ }^{3}$, F.Pühlhofer ${ }^{9}$, G.Rai ${ }^{3}$, W.Rauch ${ }^{10}$, R.Renfordt ${ }^{7}$, D.Röhrich ${ }^{7}$, H.Rothard ${ }^{7}$, K.Runge $^{8}$, A.Sandoval $^{6}$, E.Schmidt ${ }^{7}, \quad$ N.Schmit ${ }^{10}$, E.Schmoetten ${ }^{8}$,I.Schneider ${ }^{7}$,P.Seyboth ${ }^{10}{ }^{10}$,J.Seyerlein ${ }^{10}, \quad$ E.Skrzypczak ${ }^{11}$, P.Stefański ${ }^{5}$, R.Stock ${ }^{7}$, H.Ströbele ${ }^{7}$, L.Teitelbaum ${ }^{3}, \quad$ S.Tonse ${ }^{3}$, G.Vassileiadis $^{1}$,

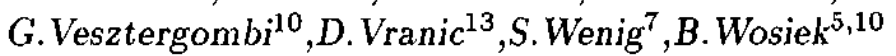

1)Physics Dept.,Univ.of Athens 2)INFN,Sez.Bari and Dipartimento di Fisica, Univ. di Bari 3)Lawrence Berkeley Lab. 4)CERN 5)Inst.of Nuclear Physics,Cracow 6)GSI Darmstadt 7)Fachbereich Physik, Univ.Franfurt 8)Fakultät für Physik, Univ.Freiburg 9)Fachbereich Physik,Univ.Marburg 10)Max-Planck-Institut für Physik, München 11)Inst.of Experimental Physics,Univ. of Warsaw 12)Institute of Nuclear Studies, Warsaw 13)Rudjer Boskovic Inst.,Zagreb

\begin{abstract}
Multiplicity distributions of negatively charged particles have been studied in restricted phase space intervals for central $S+S, O+A u$ and $S+A u$ collisions at $200 \mathrm{GeV} /$ nucleon. It is shown that multiplicity distributions are well described by a negative binomial form irrespectively of the size and dimensionality of phase space domain. A clan structure analysis reveals interesting similarities between complex nuclear collisions and a simple partonic shower. The lognormal distribution agrees reasonably well with the multiplicity data in large domains, but fails in the case of small intervals. No universal scaling function was found to describe the shape of multiplicity distributions in phase space intervals of varying size.
\end{abstract}





\section{Introduction}

Recently the moments of multiplicity distributions in phase-space domains of variable size have been extensively studied for different high energy collisions [1]. The basic motivation for this work was to reveal the intermittency phenomenon, i.e.nonstatistical fluctuations in the particle density distributions. The numerous experimental results which became available during the last years indeed confirmed the intermittent or fractal behaviour of particle spectra [1]. However, many questions still remain unanswered and require further both experimental and theoretical studies. One of the problems we want to address in this paper concerns the description of the shape of multiplicity distributions. Studying the multiplicity distribution itself may provide more complete information about the fluctuations than the analysis of its moments. In particular, relations between moments of different orders can be obtained.

It has been shown [2] that in a simple self-similar cascade model a higher order factorial moment, $F_{q}$, depends linearly on $F_{2}$ in a $\log$-log plot:

$$
\log F_{q} \approx\left(\varphi_{q} / \varphi_{2}\right) \log F_{2},
$$

where $\varphi_{q}$ is the exponent in the power-law dependence of $F_{q}$ on the number of phase space boxes. Recent experimental results are consistent with this relation [3]. Furthermore the slope ratios $\varphi_{q} / \varphi_{2}$ are observed to be approximately independent of the collision process and the dimensionality of the phase space domain. In consequence, all multiplicity distributions in different phase space intervals should have a rather simple form.

In this paper we concentrate on the analysis of the multiplicity distributions of particles produced in central nucleus-nucleus collisions. In central nuclear collisions the fluctuations are in general smaller than in more elementary processes, and are dominated by the short range two particle correlations. Thus, one can expect to find for nuclear collisions even simpler relations between the moments of different orders than that given by eq.(1). So far the multiplicity distributions in nuclear collisions have been analysed only in one dimensional phase space intervals [4]. Here, we extend the study of multiplicity distributions in nucleus-nucleus collisions also to two- and three-dimensional phase space domains.

\section{Experimental determination of the multiplic- ity distributions}

We have measured the multiplicity distributions $(M D s)$ of particles produced in central $S+S, O+A u$ and $S+A u$ interactions at $200 \mathrm{GeV}$ per nucleon in the streamer chamber of the NA35 experiment [5]. Two triggers were used in the experiment to select central collisions. For $S+S$ and $O+A u$ collisions the forward energy. trigger was applied. It selected events with an energy deposited in the projectile 
fragmentation region smaller than a given value. The other trigger, used for $S+A u$ interactions, was obtained from the transverse energy signal. It selected events in which the total transverse energy deposited in the mid-rapidity region exceeded a preset threshold. The selected samples represent $2.6 \%$ of $\sigma_{\text {tot }}$ for $S+S$ collisions, $6 \%$ of $\sigma_{t o t}$ for $O+A u$ collisions and $1.3 \%$ of $\sigma_{t o t}$ for $S+A u$ interactions.

The study is restricted to negatively charged secondaries only, produced in a limited phase-space region in which the detection efficiency is the best. The applied cuts are $\Delta y=2(1<y<3)$ in rapidity and $\Delta \phi=\pi$ in azimuthal angle for all data sets. The transverse momentum $p_{T}$ ranges from 0.005 to $2.0 \mathrm{GeV} / \mathrm{c}$.

Similarly to intermittency studies, we have analysed the $M D s$ in phase space boxes of different dimensions, namely in one dimension $(1 D)(y)$, two dimensions $(2 D)(y, \phi)$ and three dimensions $(3 D)\left(y, \phi, \ln p_{T}\right)$. In order to eliminate any effects caused by the variable shape of the single particle distributions we have used the variables $\tilde{y}, \tilde{\phi}, \ln \tilde{p}_{T}$ obtained by an integral transformation [6] for which the single particle density distributions $(d n / d \tilde{y}, e t c$.$) are flat.$

The overall numbers of analysed events are low $(2856,463$ and 270 events for $S+S, O+A u$ and $S+A u$ collisions respectively) giving rather large statistical errors. These errors were reduced by combining the data from all intervals of a given size. In this way, if the full analysed phase-space has been divided into $M$ boxes of the same size, the effective number of events (or number of entries to the $M D$ histogram ) is increased. by a factor of $M$ providing a significant reduction of the statistical errors for small intervals (large $M$ ). For uniform particle density distributions this procedure is believed to be justified. An additional check has been performed by comparing the averages $(\bar{n})$ and dispersions $(D)$ of the $M D s$ in a single interval of size $\delta$ with those obtained for the distributions averaged over all intervals of that size. They agreed with each other within the statistical errors ( which were of course large for the single interval).

In order to adequately reproduce the shape of the $M D s$ at least 5 non-zero entries $\left(P_{n_{-}} \neq 0\right)$ were required. This limits the maximal number $M$ of subdivisions. For very large $M$ (the actual value depends on the collision process) only $P_{0}, P_{1}$ and $P_{2}$ are non-zero, and reliable fits are not possible.

\section{Analysis of the shape of multiplicity distribu- tions}

In the case of independent emission of particles the multiplicity distribution should follow a Poisson distribution. In Figs 1 the second normalized cumulant moment

$$
K_{2}=\left(D^{2}-\bar{n}\right) / \bar{n}^{2}
$$

is presented for $1 D, 2 D$ and $3 D$ as a function of the number $M$ of subdivisions of the full phase space into smaller boxes. It is clearly seen that in particular at larger $M$ the multiplicity distributions differ from Poissonian $\left(K_{2}=0\right)$. For a given value 
of $M$ (i.e. for the same average multiplicity per box for a given collision process) the deviation from Poissonian increases with increasing dimensionality of the phase space cell.

In the following we concentrate on two parametrizations for the $M D s$ :

- the negative binomial distribution $(N B D)$, and

- the lognormal distribution $(L N D)$.

The $N B D$ was proposed in the context of multiparticle production a long time ago [8]. It provided a good parametrization for a large amount of experimental data at various energies and in different reactions, not only in the full rapidity regions but also in small rapidity windows [9]. The distribution is given by

$$
P_{n}(\bar{n}, k)=\left(\begin{array}{c}
n+k-1 \\
n
\end{array}\right)\left(\frac{\bar{n}}{\bar{n}+k}\right)^{n}\left(\frac{k}{\bar{n}+k}\right)^{k} .
$$

It depends on two parameters: $\bar{n}$ is the average multiplicity and $1 / k$ is the so called aggregation coefficient which influences the shape of the distribution and is related to its dispersion $D$ via

$$
\frac{D^{2}}{\bar{n}^{2}}=\frac{1}{\bar{n}}+\frac{1}{k}
$$

The special attraction of the negative binomial form is associated with its intepretation in terms of a QCD cascading process. A simplified parton shower model discussed by Giovannini et al. [10] leads to the $N B$ regularity both on the partonic and the hadronic level. The clan picture which was proposed by Giovannini and Van Hove $\{11]$, relates the model parameters, i.e. the average number of clans $\left(\bar{N}_{C L}\right)$ and the average clan size $\left(\bar{n}_{C L}\right)$, with the parameters of the $N B D$ :

$$
\begin{gathered}
\bar{N}_{C L}=k \ln \left(1+\frac{\bar{n}}{k}\right), \\
\bar{n}_{C L}=\bar{n} / \bar{N}_{C L} .
\end{gathered}
$$

We have fitted the multiplicity distributions in different phase space domains to the $N B D$. The results of the fits are summarized in Tables 1, 2 and 3 for $S+S, O+A u$ and $S+A u$ collisions respectively. Examples of the multiplicity distributions together with the fitted $N B D$ are shown in Figures 2,3 and 4 . In all cases the distributions are found to be well described by the negative binomial form. A similar conclusion was also obtained from the one-dimensional analysis of semi-central nucleus-nucleus collisions [4].

Another popular choice for the parametrization of multiplicity distributions is the lognormal distribution $(L N D)$. This distribution can be obtained in a wide range of cascade-like processes for which the final particle density in a phase-space domain is a product rather than a sum of the independent particle densities from the individual steps in the cascade $[12,13,14]$. Recently the lognormal distribution 
has been successfully compared to the $M D$ of charged particles produced in the full phase-space in $e^{+} e^{-}[15]$ and $p p$ collisions $[14,16]$. So far no comparison with the nuclear data has been performed.

The lognormal distribution of the continuous variable $x$ has the form $(\ln x$ is distributed normally)

$$
f(x)=\frac{N}{\sqrt{2 \pi \sigma^{2}}} \frac{1}{x} \exp \left(-\frac{(\ln x-\mu)^{2}}{2 \sigma^{2}}\right) .
$$

The discrete probability distribution $P_{n}$ is defined through

$$
P_{n}(\mu, \sigma)=\int_{n}^{n+1} f(x) d x
$$

We have tried to fit our $M D s$ by this two-parameter lognormal form. Only for multiplicity distributions in large phase space cells for $O+A u(M=1)$ and $S+A u$ $(M \leq 4)$ collisions we obtained fits of good quality. However for these data the statistical errors are large due to the low number of analysed events, and thus the corresponding $\chi^{2}$ test is not discriminative. In all other cases the $L N D$ is not compatible with the data. The main reason for this discrepancy is that for the two-parameter $L N D$ one expects a Gaussian distribution in $\log n$, which is symmetric around $\overline{\log n}$, whereas the experimental distributions are asymmetric. This is illustrated in Fig. 5 where the multiplicity distribution, multiplied by $n_{-}$ (because of the factor $1 / x$ in eq.(7)), is plotted as a function of $\log n$ in the full interval $(M=1)$ for $S+S$ collisions. The data are seen to show an asymmetry with a longer tail at low multiplicities, and a rapid cut-off at large multiplicities. In order to obtain better fits to the $L N D$ it was proposed [12] to introduce an additional shift parameter ${ }^{1}, c$, leading to

$$
P_{n}(\mu, \sigma, c)=\int_{n}^{n+1} \frac{N}{\sqrt{2 \pi \sigma^{2}}} \frac{1}{x+c} \exp \left(-\frac{(\ln [x+c]-\mu)^{2}}{2 \sigma^{2}}\right) d x .
$$

When fitting the shape of the $M D s$ the parameters $d$ and $\hat{n}$ were used which are related to $\sigma$ and $\mu$ through

$$
\begin{gathered}
\hat{n}=\exp \left(\mu+\sigma^{2} / 2\right)-c, \\
d=(\hat{n}+c) \sqrt{\exp \left(\sigma^{2}\right)-1}
\end{gathered}
$$

They can be identified as the mean and the width of the continuous distribution respectively. The mean of the discrete distribution $P_{n}$ is $\bar{n}=\hat{n}-0.5$. The corresponding $\chi^{2} / N D F$ values, obtained for the 3-parameter $L N$ fits, are listed in the last columns of Tables 1,2 and 3. The fitted distributions are represented by the dashed lines in Figs. 2,3 and 4.The $L N D$ with the additional parameter $c$ describes the $M D s$ satisfactorily, but only for large phase-space cells. For smaller cells (large $M$ ), the same discrepancy as for the 2-parameter $L N D$ remains: The log-normal form underestimates the low multiplicity tail, and overestimates the tail of large multiplicities.

\footnotetext{
${ }^{1}$ Actually this form was fitted to $p p$ and $e^{+} e^{-}$data $[15,16,14]$ in full phase space.
} 


\section{Scaling of multiplicity distributions}

Scaling properties of $M D s$ in the full phase space were long ago established in $p p$ inelastic collisions as well as in $e^{+} e^{-}$interactions in a wide range of primary energies. $M D s$ measured at different energies were found to obey the KNO (or KNO-G [12]) scaling, i.e.

$$
P(n)=\frac{1}{\bar{n}} \psi(n / \bar{n}),
$$

where $\psi(n / \bar{n})$ is the energy independent scaling function.

Here, we want to investigate whether the $M D s$ measured in different phase space boxes also exhibit some scaling when varying the box size, and how this scaling depends on the collision process. Contrary to what was observed in $p p$ and $e^{+} e^{-}$ collisions, in central heavy ion interactions the linear relation between $D$ and $\bar{n}$ (Wróblewski relation [17]) turns out not to be valid. (Whereas in the $p p$ and $e^{+} e^{-}$ studies $\bar{n}$ varies with energy, here $\bar{n}$ varies with the box size.) Instead the variance itself seems to depend linearly on the average multiplicity:

$$
D^{2}=a+b \bar{n} .
$$

Indeed our multiplicity data measured in small phase space boxes of different size $(M>8)$ can be approximately fitted by Eq.(13). The results of the fits are quoted in Table 4. A similar dependence is also characteristic for a Poissonian distribution with $a=0$ and $b=1$. However, the values of the parameter $b$ obtained from fitting the data to the relation (13) as well as very large $\chi^{2}$ values obtained for $a=0, b=1$ (see last column of Table 4) once more indicate that the measured distributions differ from a Poissonian form.

The observed relation (13) between dispersion and average multiplicity has important consequences for the choice of the scaling variable. When $D$ is not proportional to the average multiplicity the natural scaling variable should be chosen as $^{2}$

$$
z=\frac{n-\bar{n}}{D}
$$

Consequently, we propose to investigate the scaling function $\psi(z)$ defined by

$$
P(n)=\frac{1}{D} \psi(z)
$$

The scaling function $\psi(z)$ has following properties:

$$
\bar{z}=0,
$$

and

$$
\sigma_{z}=1
$$

\footnotetext{
${ }^{2}$ This variable was first introduced in [18] to parametrize the multiplicity distributions in hadronic collisions at low energies.
} 
where $\sigma_{z}$ is the dispersion of $\psi(z)$. Thus by the very choice of the form (15) one eliminates the dependence of the $M D s$ on the first two moments. Hence, $\psi(z)$ is more sensitive to higher moments, i.e. finer details of the distributions.

First of all we have checked whether a unique function $\psi(z)$ can describe the $M D s$ measured in phase space boxes of varying size. The distributions $\psi(z)$ versus $z$ as calculated for different $M$ are presented in Fig. 6 . In addition also the sensitivity of $\psi(z)$ to the collision process was studied. The relevant plots are shown in Fig. 7 .

From Figs. 6 and 7 the following conclusions can be drawn:

1. Regarding the dependence on the size of the phase space domain for a given reaction (Fig.6), a deviation from scaling behaviour is observed at larger $z$ values, the high multiplicity tail increasing with decreasing size of the domain.

2. Regarding the dependence on the reaction type for a fixed size of the phase space region (Fig. 7 ), only for large domains $(M \leq 8)$ the distributions are approximately independent of the collision process; for smaller domains the distributions deviate from each other, the large multiplicity tail being less pronounced for heavier collision systems. However, it should be pointed out that this conclusion is sensitive to the trigger selections which are not exactly the same for each analysed data sample.

\section{Discussion}

Since the $N B D$ provided a good parametrization of the measured $M D s$ in the. domains of different sizes we limit our discussion to the $N B$ results (see Tables 1,2 and 3$)$. For the $1 D$ analysis the parameter $1 / k$ is within the errors independent of the size of the rapidity window (see also [19]). However in the case of $2 D$ and $3 D$ boxes a systematic increase of $1 / k$ with decreasing box size is observed. The increase is more pronounced for $3 D$. This means that the particle aggregation gets larger in small boxes and is higher in $3 D$ than in one dimensional boxes.

The clan structure analysis (eqs. (5), (6) ) reveals the following regularities (see Fig.8): The average number of clans rapidly decreases with decreasing size of the domain, whereas the average number of particles per clan remains constant at the level of about 1 negative particle per clan. Interestingly a similar regularity was recently obtained [20] within the framework of a simplified parton- shower model for the dependence of the hadronic clan parameters on the size of the rapidity interval for fixed virtuality of the initial parton. The fact that such complex phenomena like nucleus-nucleus central collisions seem to have properties similar to elementary processes suggests the idea that the same dynamical mechanism (QCD parton showerformation) may be responsible for multiparticle production in different high energy collisions.

For the $N B D$ the normalized factorial moments $F_{q}$ are governed by the param- 
eter $1 / k$ and can be derived from the following formula

$$
F_{q}=\left(1+\frac{q-1}{k}\right) F_{q-1} .
$$

Thus all moments of the order $q>2$ can be calculated from $F_{2}$. In Fig. $9 \log F_{q} v s . \log F_{2}$ plots are presented to compare the $N B D$ expectations and the moments obtained directly from the data. The data points are consistent with the $N B$ relation ${ }^{3}$ (18) independently of the reaction type. Note that in the studied range of $F_{2}$ values this relation is approximately linear and consistent with the universal relation (1). However it was noted [21] that for larger values of $F_{2}$ neither the $N B$ nor the $L N$ prediction agrees with a linear relation. In [21] other forms of the multiplicity distribution are discussed which are compatible with a self-similar cascade model with a multiplicative formula for particle densities. They may provide a better description of the data in case of large multiplicity fluctuations. We have shown here that for nuclear data, where the fluctuations are small and multiplicity distributions do not exhibit a large high multiplicity tail, the popular $N B$ form successfully describes the data.

\section{Summary}

The negative particle multiplicity distributions have been studied for central $S+S$, $O+A u$ and $S+A u$ collisions at $200 \mathrm{GeV}$ per nucleon, in phase space cells of different size and dimensionality.

It has been shown that the $M D s$ differ from Poissonian, the largest deviations being observed in the case of three-dimensional cells. On the other hand the multiplicity data are successfully described by the negative binomial form, independently of the size of the phase space cell as well as its dimensionality. The negative binomial distribution parameter $1 / k$ is (within the errors) independent of the width of the rapidity interval. However it increases with decreasing cell size for two- and three-dimensional cells, indicating that the $M D s$ are broader in smaller cells. The interpretation in terms of the clan cascading picture leads to an interesting similarity between the dependence of the model parameters on the cell size in nuclear collisions and simple partonic cascades. The linear relation between $\log F_{q}$ and $\log F_{2}$ is consistent with the $N B$ expectations in the available range of $F_{2}$ values.

The lognormal distribution describes the multiplicity data only in large cells; for smaller domains it is definitely not compatible with the data.

There is no universal scaling function which can describe the multiplicity distributions measured in phase space regions of different size.

\footnotetext{
${ }^{3}$ This was obviously expected on the base of the good quality of the $N B$ fits.
} 


\section{Acknowledgement}

We wish to thank the CERN accelerator and experimental area groups for providing excellent working conditions. We are very grateful to our scanning and measuring crews at Frankfurt, München, Warsaw and Zagreb for their careful work. This work was supported by the Bundesministerium für Forschung und Technologie,FRG, by the US Department of Energy (Contracts DE-AC03-76SF00098 and DE-AC02-76CH00016), by the Hellenic Secretariat for Research and Technology, by the Comission of the European Communities in cooperation with the Ministry for Science of Croatia and by Polish Government research grant Nr. 204369101. 


\section{References}

[1] Eds. R.C.Hwa, W.Ochs and N.Schmitz, Proceedings of Ringberg Workshop on Multiparticle Production-Fluctuations and Fractal Structure, World Scientific, Singapore 1992

[2] W.Ochs and J.Wosiek, Phys.Lett.B214 (1988)617

[3] N.Schmitz, Proc. XXI International Symposium on Multiparticle Dynamics, Wuhan, China, p.377 (1991), World Scientific, Singapore

[4] B.Wosiek, INP report No.1533/PH,1991

[5] A.Bamberger et al., Phys.Lett.B205 (1988) 583; J.Bartke et al.,Z.Phys.C48 (1990) 191; J.Bächler et al., Z.Phys.C51 (1991) 157

[6] W.Ochs, Z.Phys.C50 (1991) 339; A.Białas and M. Gaździcki Phys.Lett.B252 (1990) 483

[7] P.Seyboth et al., Nucl.Phys.A544 (1992) 293c

[8] A.Giovannini, Lett.Nuovo Cimento 6 (1973) 514; P.Caruthers and C.C.Shih, Phys.Lett. 127B (1983) 42; P.Caruthers and C.C.Shih, Phys.Lett. 137B (1984) 425; M.Biyajima, Progr.Theor.Phys. 69 (1983) 966

[9] M.Adamus et al.,Z.Phys. C37 (1988) 215; R.E.Ansorge et al.,Z.Phys.C43 (1988) 357; N.Schmitz, Proc. Meeting on Multiparticle Dynamics (Festschrift for Léon Van Hove), La Thuile, Italy, p.25 (1989), World Scientific, Singapore

[10] A.Giovannini and L.Van Hove, Acta Phys.Pol.B19 (1988) 495, 917, 931, and Proc. XXV International Conference on High Energy Physics, Singapore, p.998 (1990), World Scientific, Singapore; A.Giovannini, Proc. XXI International Symposium on Multiparticle Dynamics, Wuhan, China, p.285 (1991), World Scientific, Singapore, and preprint DFTT 48/91 (1991)

[11] A.Giovannini and L.Van Hove, Z.Phys. C30 (1986) 391; Acta Physica Polonica B19 (1988) 405

[12] R.Szwed and G.Wrochna, Z.Phys.C29 (1985) 255

[13] R.Szwed, G.Wrochna, A.Wróblewski, Mod.Phys.Lett.A5 (1990) 1851; R.Szwed and G.Wrochna, Z.Phys. C47 (1990) 447

[14] S.Carius and G.Ingelman, Phys.Lett.B252 (1990) 647

[15] D.Decamp et al., ALEPH Collaboration, Phys.Lett.B273 (1991) 181; P.D.Acton et al., OPAL Collaboration, Z.Phys.C53 (1992) 539; P.Abreu et al., DELPHI Collaboration, Z.Phys.C52 (1991) 271 
(16] M.Gaździcki et al., Mod.Phys.Lett.A6 (1991) 981

[17] A.Wróblewski, Acta Phys.Pol.B4 (1973) 857

[18] O.Czyżewski and K.Rybicki, Nucl. Phys.B47 (1972) 633

[19] J.Bächler et al.,NA35 Collaboration, Z.Phys.C in press

[20] R.Ugoccioni and A.Giovannini, Z.Phys.C53 (1992) 239

[21] K.Fiałkowski, W.Ochs, I.Sarcevic, Z.Phys.C54 (1992) 621 


\begin{tabular}{|c|r|l|l|c||c|}
\hline dim. & $M$ & $1 / k$ & $\bar{n}$ & $\chi^{2} / N D F$ & $\chi^{2} / N D F$ for $L N D$ \\
\hline \hline $1 \mathrm{D}$ & 1 & $0.0100(17)$ & $20.84(10)$ & $22.8 / 32$ & $13.6 / 31$ \\
& 2 & $0.0127(21)$ & $10.40(5)$ & $37.2 / 24$ & $24.5 / 23$ \\
& 4 & $0.0132(27)$ & $5.20(2)$ & $25.2 / 17$ & $20.7 / 16$ \\
& 8 & $0.0199(39)$ & $2.60(1)$ & $11.5 / 10$ & $13.8 / 9$ \\
& 16 & $0.0185(53)$ & $1.300(5)$ & $1.6 / 8$ & $153 / 7$ \\
& 32 & $0.0175(75)$ & $0.6497(27)$ & $8.5 / 6$ & $441 / 5$ \\
& 64 & $0.0215(145)$ & $0.3251(18)$ & $0.3 / 4$ & $220 / 3$ \\
& 128 & $0.0232(148)$ & $0.1625(7)$ & $1.1 / 4$ & $209 / 3$ \\
& 256 & $0.0341(206)$ & $0.0813(3)$ & $1.8 / 3$ & $115 / 2$ \\
\hline 2D & 4 & $0.0148(26)$ & $5.192(22)$ & $32.1 / 17$ & $28.8 / 16$ \\
& 9 & $0.0285(41)$ & $2.310(10)$ & $7.5 / 10$ & $23.2 / 9$ \\
& 16 & $0.0283(52)$ & $1.300(5)$ & $10.5 / 8$ & $201 / 7$ \\
& 36 & $0.0435(81)$ & $0.5778(24)$ & $2.5 / 5$ & $467 / 4$ \\
& 64 & $0.0468(105)$ & $0.3251(13)$ & $0.1 / 4$ & $409 / 3$ \\
& 121 & $0.0525(145)$ & $0.1720(7)$ & $0.8 / 3$ & $236 / 2$ \\
& 196 & $0.0654(106)$ & $0.1061(4)$ & $0.03 / 3$ & $148 / 2$ \\
& 256 & $0.0705(209)$ & $0.0813(3)$ & $2.2 / 3$ & $126 / 2$ \\
\hline $3 \mathrm{D}$ & 8 & $0.0288(39)$ & $2.601(11)$ & $8.2 / 10$ & $15.2 / 9$ \\
& 27 & $0.0542(72)$ & $0.7702(32)$ & $13.6 / 7$ & $459 / 6$ \\
& 64 & $0.0808(108)$ & $0.3250(13)$ & $3.1 / 5$ & $456 / 4$ \\
& 125 & $0.0899(152)$ & $0.1664(7)$ & $2.1 / 4$ & $228 / 3$ \\
& 216 & $0.1648(174)$ & $0.0963(4)$ & $0.7 / 3$ & $176 / 2$ \\
& 343 & $0.1943(263)$ & $0.0606(2)$ & $1.3 / 3$ & $88.7 / 3$ \\
\hline
\end{tabular}

Table 1: Results of fits of the $N B D$ to the negative multiplicity distributions in $S+S$ central collisions. In the last column the $\chi^{2} / N D F$ values of fits to the 3-parameter $L N D$ are given. The numbers in parentheses give the uncertainty in the last digits of the associated parameters. 


\begin{tabular}{|c|r|l|l|c||c|}
\hline dim. & $M$ & $1 / k$ & $\bar{n}$ & $\chi^{2} / N D F$ & $\chi^{2} / N D F f$ or $L N D$ \\
\hline \hline 1D & 1 & $0.0233(43)$ & $34.49(39)$ & $30.3 / 33$ & $30.3 / 32$ \\
& 2 & $0.0209(41)$ & $16.96(16)$ & $26.5 / 29$ & $20.3 / 28$ \\
& 4 & $0.0274(51)$ & $8.427(75)$ & $18.2 / 20$ & $15.1 / 19$ \\
& 8 & $0.0249(60)$ & $4.209(36)$ & $12.6 / 12$ & $16.5 / 11$ \\
& 16 & $0.0250(77)$ & $2.103(16)$ & $11.7 / 9$ & $20.2 / 8$ \\
& 32 & $0.0369(119)$ & $1.054(9)$ & $3.4 / 6$ & $70.6 / 5$ \\
& 64 & $0.0270(154)$ & $0.5269(32)$ & $1.2 / 4$ & $138 / 3$ \\
& 128 & $0.0364(224)$ & $0.2637(16)$ & $0.3 / 4$ & $99.5 / 3$ \\
& 256 & $0.0134(315)$ & $0.1318(8)$ & $1.3 / 4$ & $55.7 / 3$ \\
\hline 2D & 4 & $0.0304(49)$ & $8.412(75)$ & $17.8 / 19$ & $13.4 / 18$ \\
& 9 & $0.0408(71)$ & $3.742(32)$ & $11.3 / 13$ & $10.5 / 12$ \\
& 16 & $0.0557(95)$ & $2.104(18)$ & $11.5 / 9$ & $14.3 / 8$ \\
& 36 & $0.0638(128)$ & $0.9371(77)$ & $2.5 / 6$ & $90.3 / 5$ \\
& 64 & $0.0824(172)$ & $0.5276(35)$ & $0.1 / 4$ & $106 / 3$ \\
& 100 & $0.0814(206)$ & $0.3376(21)$ & $1.8 / 3$ & $105 / 2$ \\
& 169 & $0.0894(266)$ & $0.1994(16)$ & $4.3 / 4$ & $83.4 / 3$ \\
& 289 & $0.0703(322)$ & $0.1166(9)$ & $0.2 / 3$ & $54.3 / 2$ \\
& 400 & $0.0678(411)$ & $0.0842(7)$ & $0.3 / 3$ & $25.1 / 2$ \\
\hline 3D & 8 & $0.0468(70)$ & $4.216(37)$ & $10.9 / 13$ & $10.2 / 12$ \\
& 27 & $0.0877(115)$ & $1.249(11)$ & $2.7 / 8$ & $60.3 / 7$ \\
& 64 & $0.1226(171)$ & $0.5271(36)$ & $3.4 / 5$ & $134 / 4$ \\
& 125 & $0.1228(229)$ & $0.2696(22)$ & $6.0 / 4$ & $123 / 3$ \\
& 216 & $0.1687(299)$ & $0.1560(12)$ & $4.7 / 3$ & $108 / 2$ \\
& 343 & $0.2117(394)$ & $0.0984(6)$ & $1.6 / 3$ & $22.0 / 2$ \\
& 512 & $0.2335(480)$ & $0.0659(5)$ & $3.1 / 3$ & $39.5 / 2$ \\
& 1000 & $0.3488(700)$ & $0.0337(2)$ & $2.4 / 3$ & $22.4 / 2$ \\
\hline
\end{tabular}

Table 2: Results of fits of the $N B D$ to the negative multiplicity distributions in $O+A u$ central collisions. In the last column the $\chi^{2} / N D F$ values of fits to the 3 -parameter $L N D$ are given. The numbers in parentheses give the uncertainty in the last digits of the associated parameters. 


\begin{tabular}{|c|c|c|c|c|c|}
\hline dim. & $\bar{M}$ & $1 / k$ & $\bar{n}$ & $\chi^{2} / N D F$ & $\chi^{2} / N D F$ for $L N D$ \\
\hline \multirow[t]{9}{*}{$1 \mathrm{D}$} & $\overline{1}$ & $0.0009(22)$ & $65.30(51)$ & $63.9 / 51$ & $63.4 / 50$ \\
\hline & 2 & $0.0048(31)$ & $32.23(26)$ & $47.5 / 40$ & $44.6 / 39$ \\
\hline & 4 & $0.0057(33)$ & $16.19(9)$ & $27.7 / 31$ & $25.5 / 30$ \\
\hline & 8 & $0.0112(48)$ & $8.084(50)$ & $24.2 / 17$ & $15.1 / 16$ \\
\hline & 16 & $0.0259(64)$ & $4.026(32)$ & $5.8 / 16$ & $3.4 / 15$ \\
\hline & 32 & $0.0231(77)$ & $2.007(16)$ & $8.9 / 11$ & $20.8 / 10$ \\
\hline & 64 & $0.0111(112)$ & $1.006(7)$ & $1.5 / 6$ & $68.8 / 5$ \\
\hline & 128 & $0.0086(153)$ & $0.5028(30)$ & $2.4 / 5$ & $141 / 4$ \\
\hline & 256 & $0.0217(213)$ & $0.2515(15)$ & $0.7 / 3$ & $97.4 / 2$ \\
\hline \multirow[t]{10}{*}{$2 \mathrm{D}$} & $\overline{4}$ & $0.0093(31)$ & $16.14(13)$ & $21.7 / 29$ & $18.6 / 28$ \\
\hline & 9 & $0.0112(47)$ & $7.162(56)$ & $21.9 / 18$ & $12.6 / 17$ \\
\hline & 16 & $0.0221(58)$ & $4.021(32)$ & $10.0 / 12$ & $15.6 / 11$ \\
\hline & 36 & $0.0304(83)$ & $1.785(14)$ & $10.9 / 9$ & $27.9 / 8$ \\
\hline & 64 & $0.0279(109)$ & $1.005(8)$ & $8.8 / 6$ & $94.1 / 5$ \\
\hline & 100 & $0.0430(135)$ & $0.6435(40)$ & $1.9 / 5$ & $168 / 4$ \\
\hline & 169 & $0.0657(190)$ & $0.3805(29)$ & $3.7 / 4$ & $101 / 3$ \\
\hline & 289 & $0.0637(239)$ & $0.2225(13)$ & $1.4 / 3$ & $70.0 / 2$ \\
\hline & 484 & $0.1055(294)$ & $0.1328(10)$ & $2.5 / 3$ & $97.7 / 2$ \\
\hline & 841 & $0.0519(415)$ & $0.0764(4)$ & $2.3 / 3$ & $13.9 / 2$ \\
\hline \multirow[t]{9}{*}{$3 D$} & 8 & $0.0236(47)$ & $8.041(67)$ & $25.0 / 19$ & $15.0 / 18$ \\
\hline & 27 & $0.0510(75)$ & $2.377(19)$ & $13.3 / 10$ & $21.9 / 9$ \\
\hline & 64 & $0.0789(120)$ & $1.006(8)$ & $3.4 / 6$ & $80.9 / 5$ \\
\hline & 125 & $0.1177(160)$ & $0.5148(33)$ & $4.9 / 5$ & $179 / 4$ \\
\hline & 216 & $0.1447(213)$ & $0.2979(18)$ & $1.0 / 4$ & $119 / 3$ \\
\hline & 343 & $0.1843(278)$ & $0.1877(11)$ & $0.1 / 3$ & $68.1 / 2$ \\
\hline & 512 & $0.1568(323)$ & $0.1258(9)$ & $0.2 / 3$ & $60.4 / 2$ \\
\hline & 729 & $0.2685(421)$ & $0.0881(7)$ & $0.3 / 3$ & $29.1 / 2$ \\
\hline & 1000 & $0.3144(489)$ & $0.0643(4)$ & $1.3 / 3$ & $26.3 / 2$ \\
\hline
\end{tabular}

Table 3: Results of fits of the $N B D$ to the negative multiplicity distributions in $S+A u$ central collisions. In the last column the $\chi^{2} / N D F$ values of fits to the 3-parameter $L N D$ are given. The numbers in parentheses give the uncertainty in the last digits of the associated parameters. 


\begin{tabular}{|r|c|c|c|c|}
\hline Sample & $a$ & $b$ & $\chi^{2} / N D F$ & $\chi^{2} /$ N DF forPoiss. \\
\hline \hline S+S ,1D & $-0.0009(3)$ & $1.0134(25)$ & $5.3 / 4$ & $33 / 4$ \\
O+Au,1D & $-0.0050(10)$ & $1.0384(63)$ & $4.5 / 4$ & $39 / 4$ \\
S+Au,1D & $-0.0103(27)$ & $1.0411(82)$ & $6.2 / 4$ & $38 / 4$ \\
\hline S+S ,2D & $-0.0020(2)$ & $1.0264(24)$ & $6.0 / 5$ & $90 / 5$ \\
O+Au,2D & $-0.0042(5)$ & $1.0495(46)$ & $8.3 / 6$ & $109 / 6$ \\
S+Au,2D & $-0.0024(4)$ & $1.0345(40)$ & $2.5 / 7$ & $51 / 7$ \\
\hline S+S ,3D & $-0.0011(2)$ & $1.0278(24)$ & $5.1 / 3$ & $47 / 3$ \\
O+Au,3D & $-0.0008(1)$ & $1.0377(30)$ & $11.2 / 5$ & $87 / 5$ \\
S+Au,3D & $-0.0051(3)$ & $1.0685(37)$ & $7.1 / 6$ & $280 / 6$ \\
\hline
\end{tabular}

Table 4: Results of fits to the relation (13). In the last column the $\chi^{2} / N D F$ values for fixed parameters $a=0$ and $b=1$ are given. The numbers in parentheses give the uncertainty in the last digits of the associated parameters. 


\section{Figure captions}

Fig. 1 The second normalized cumulant moment $K_{i}$ as a function of the number $M$ of subdivisions in one,two and three phase space dimensions for $S+S$, $O+A u$ and $S+A u$ collisions.

Fig.2 Multiplicity distributions of negative particles for $S+S$ collisions in phase space intervals of different size and dimensionality. The solid (dashed) lines show the $N B(3$-parameter $L N)$ fits to the data points.

Fig.3 The same as in Figs.2 but for $O+A u$ collisions.

Fig.4 The same as in Figs. 2 but for $S+A u$ collisions.

Fig.5 Probability distribution for $S+S$ interactions in the full phase space region $(M=1)$. Dashed line represents the best fitted two-parameter lognormal distribution.

Fig.6.The scaling function $\psi(z)$ as determined from multiplicity distributions for the three reactions, for three different box sizes in a) $1 \mathrm{D}$, b) $2 \mathrm{D}$ and c) 3D (see text).

Fig.7.The scaling function $\psi(z)$ for three different box sizes, for the three reactions in a) $1 \mathrm{D}, \mathrm{b}) 2 \mathrm{D}$ and c) $3 \mathrm{D}$ (see text).

Fig.8. The average number of clans $\left(\bar{N}_{C L}\right)$ and the average clan size $\left(\bar{n}_{C L}\right)$ for the three reactions as a function of the number $M$ of subdivisions in a) $1 \mathrm{D}, \mathrm{b}) 2 \mathrm{D}$ and c) $3 \mathrm{D}$ (see text).

Fig.9 $\log F_{q}$ vs $\log F_{2}$ for $q=3,4$ in 3 dimensions for the three reactions. The solid lines represent the negative binomial expectations. (The horizontal error bars are omitted for clarity of presentation.) 

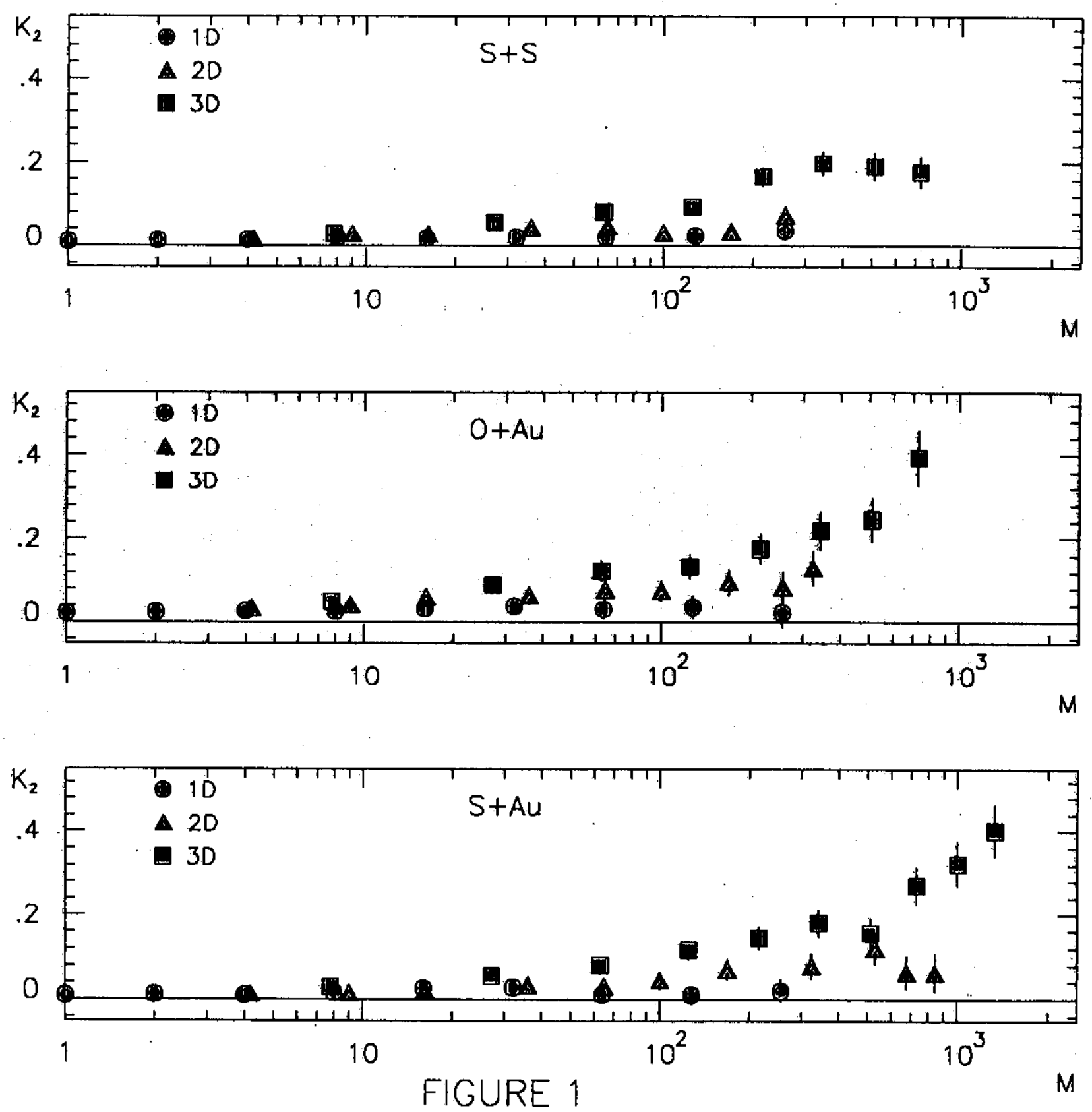

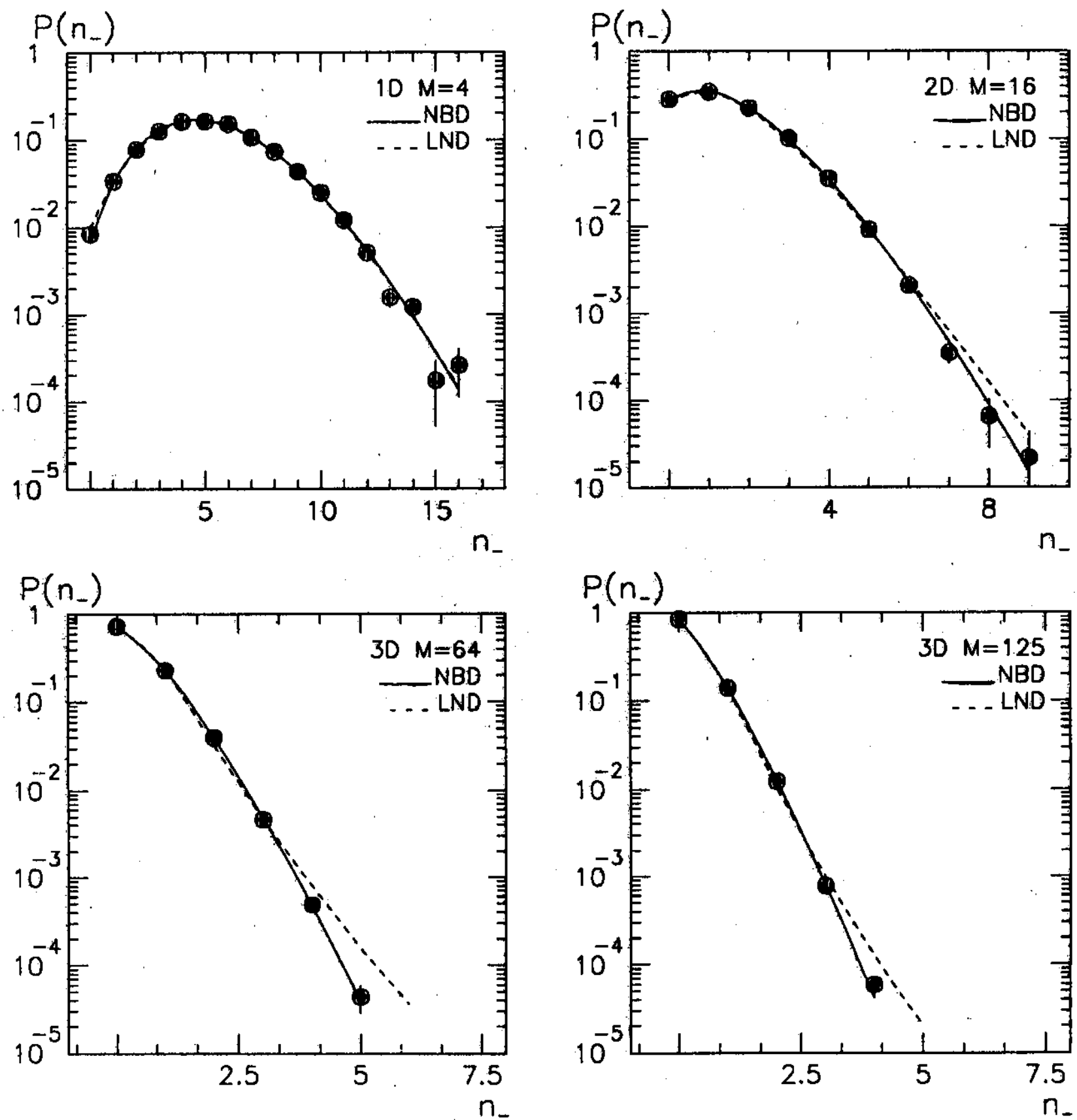

FIGURE 2 

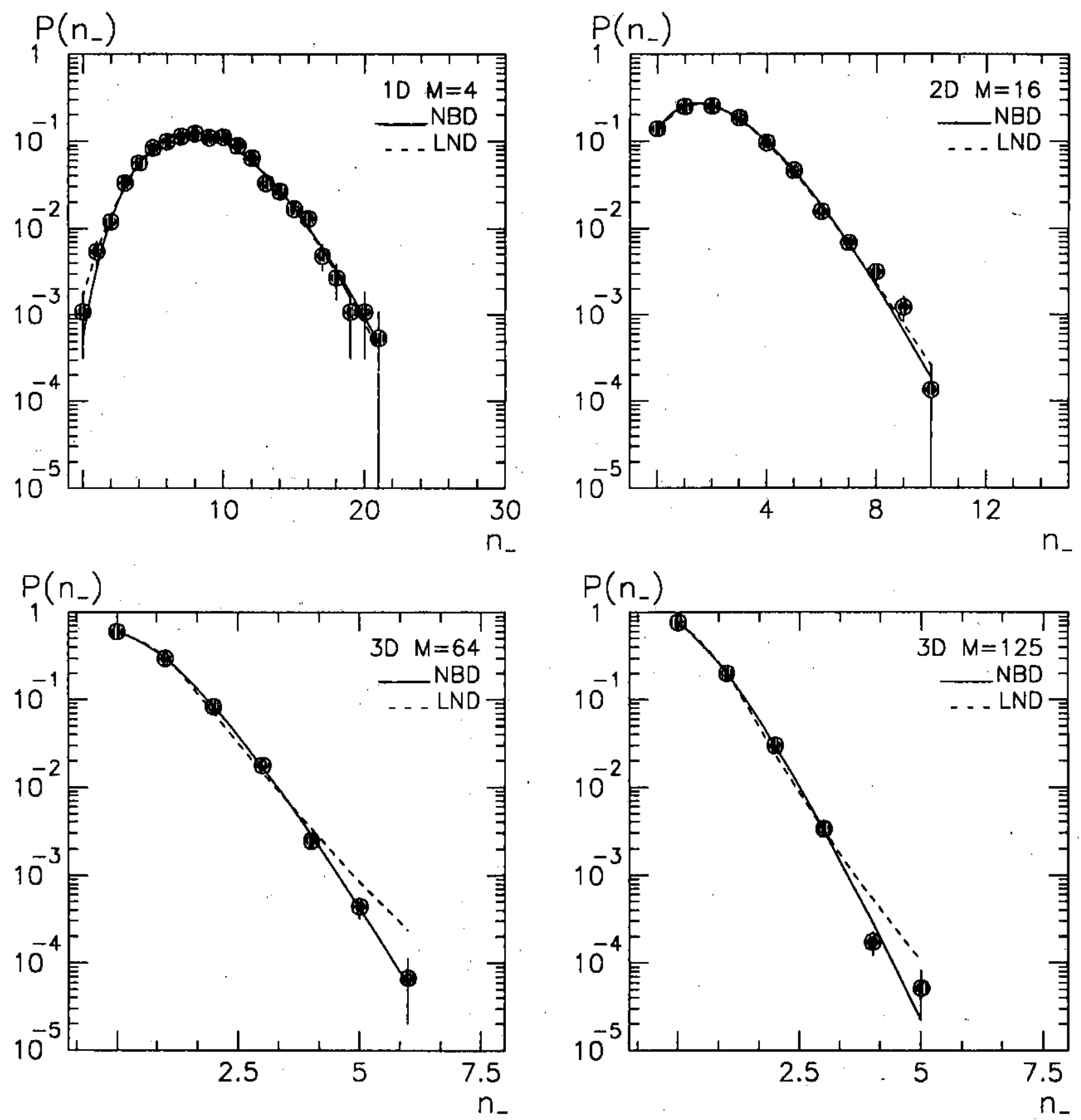

FIGURE 3 

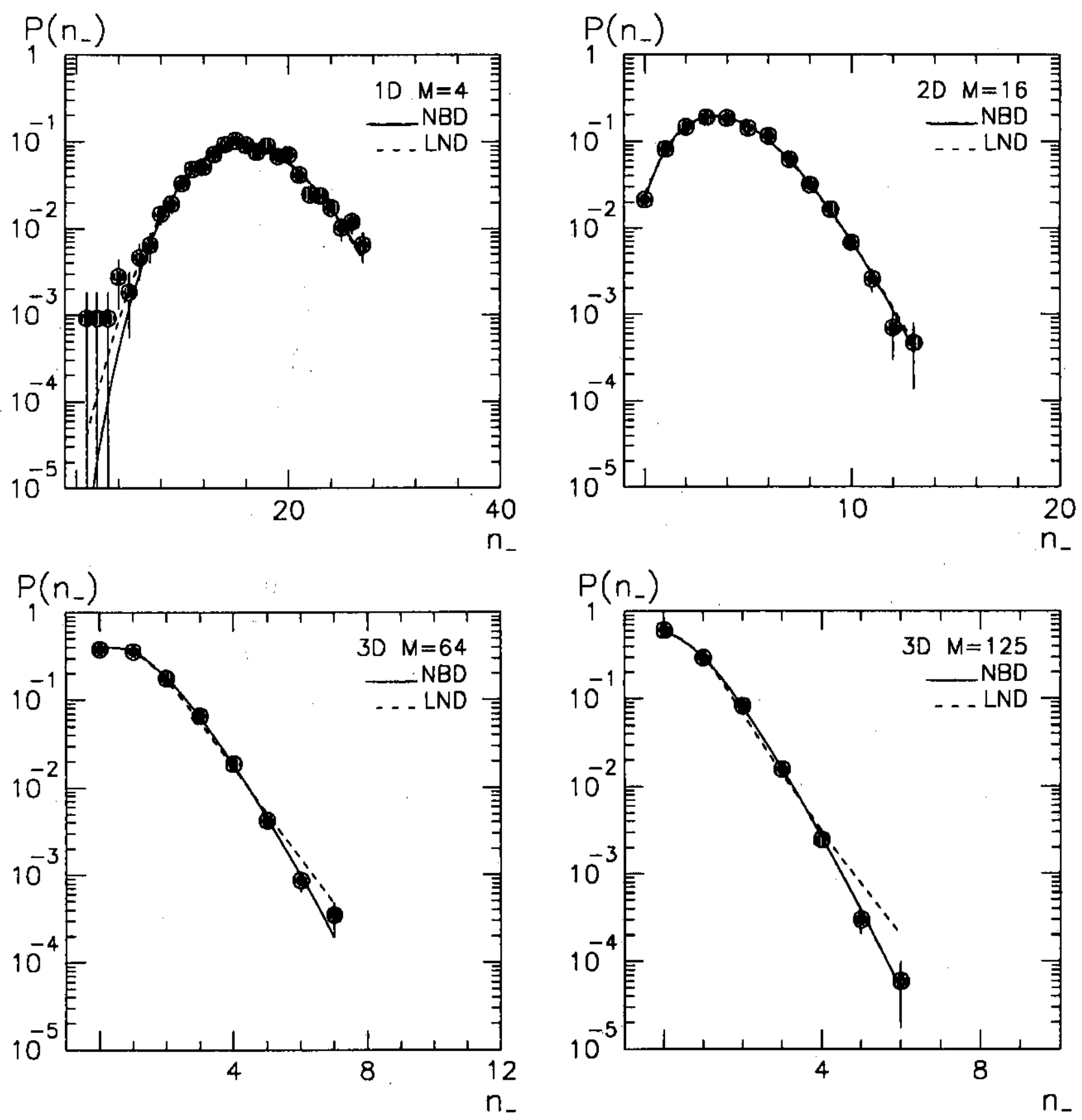

FIGURE 4 


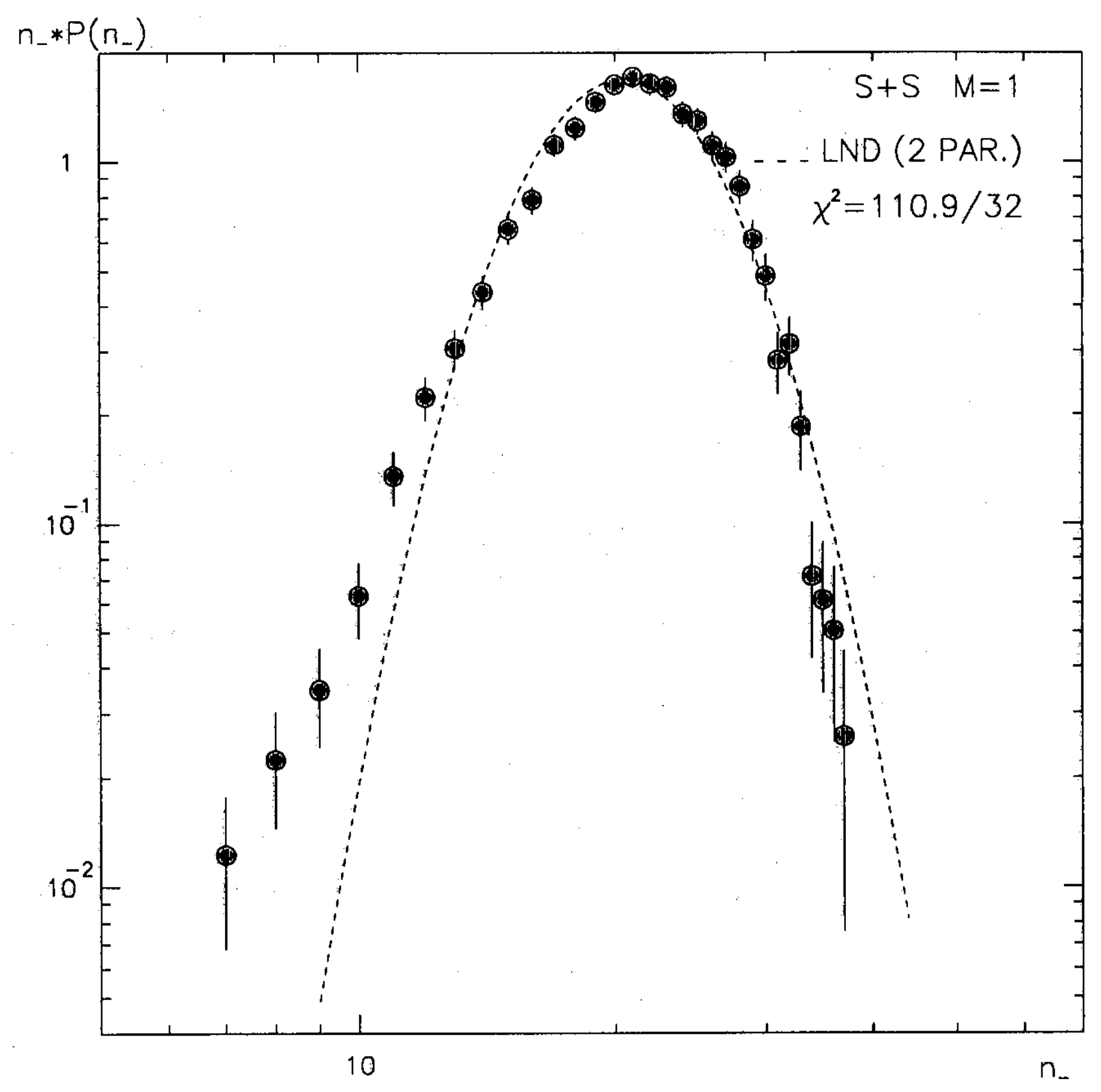

FIGURE 5 

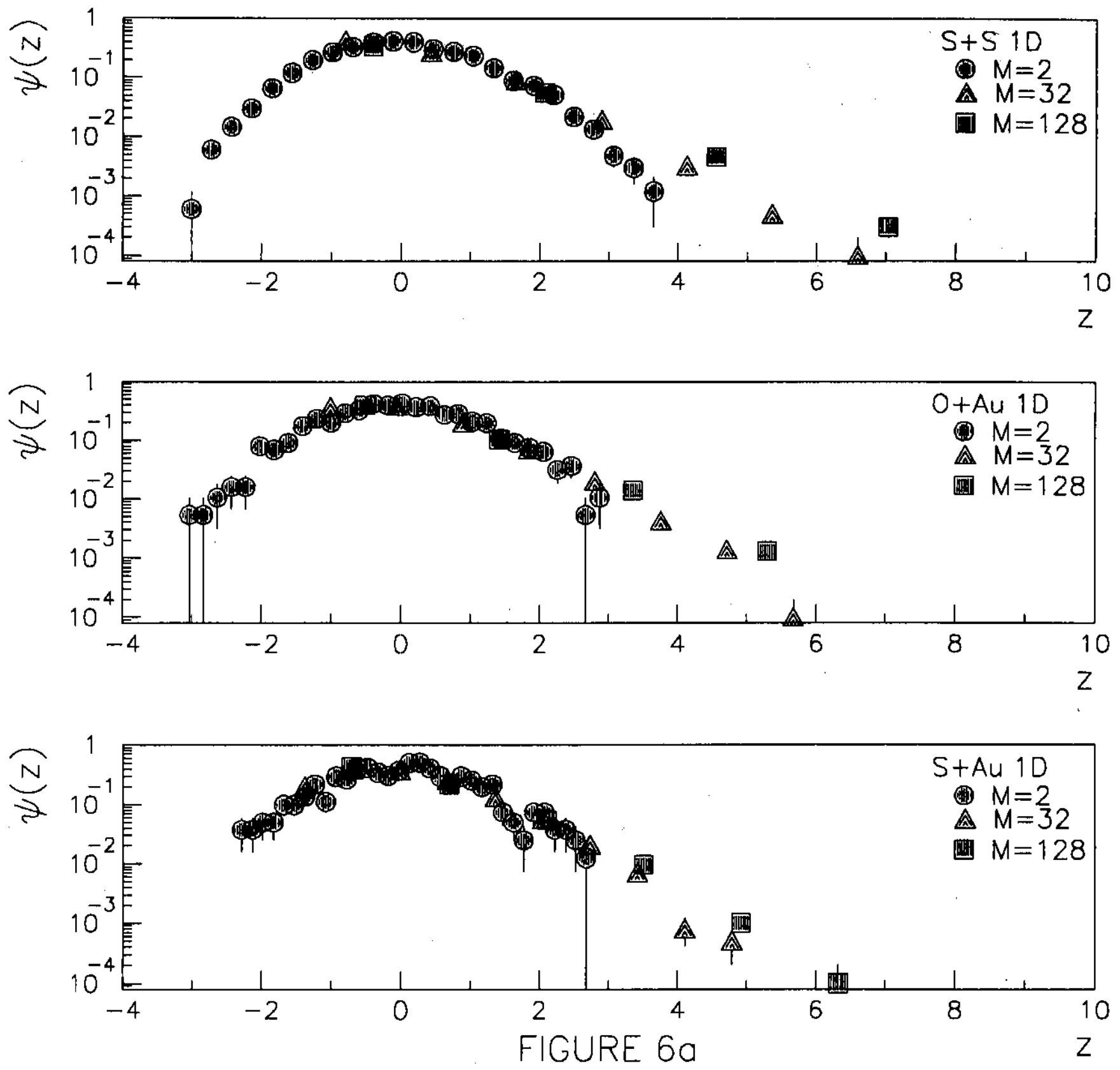

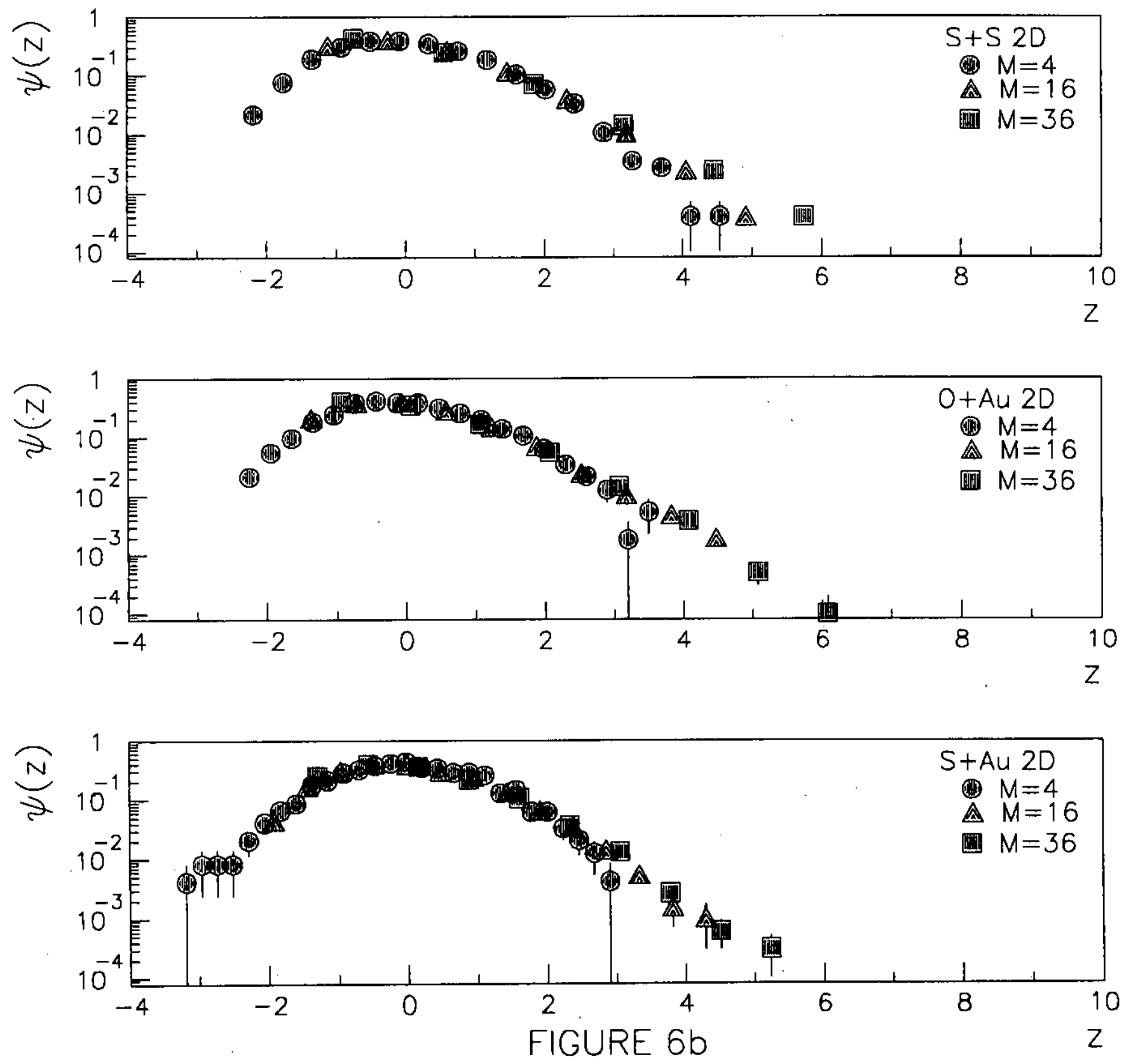

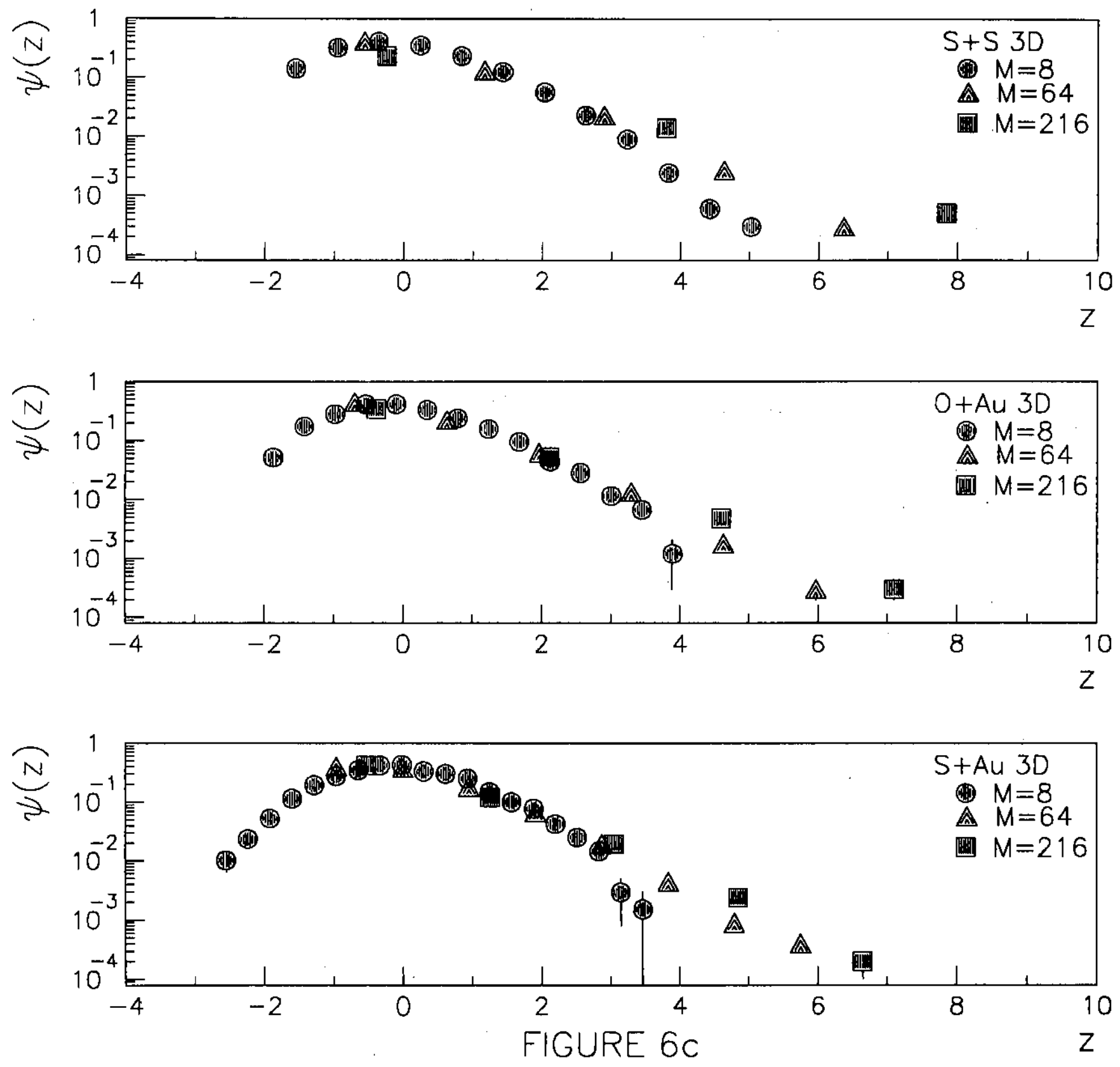

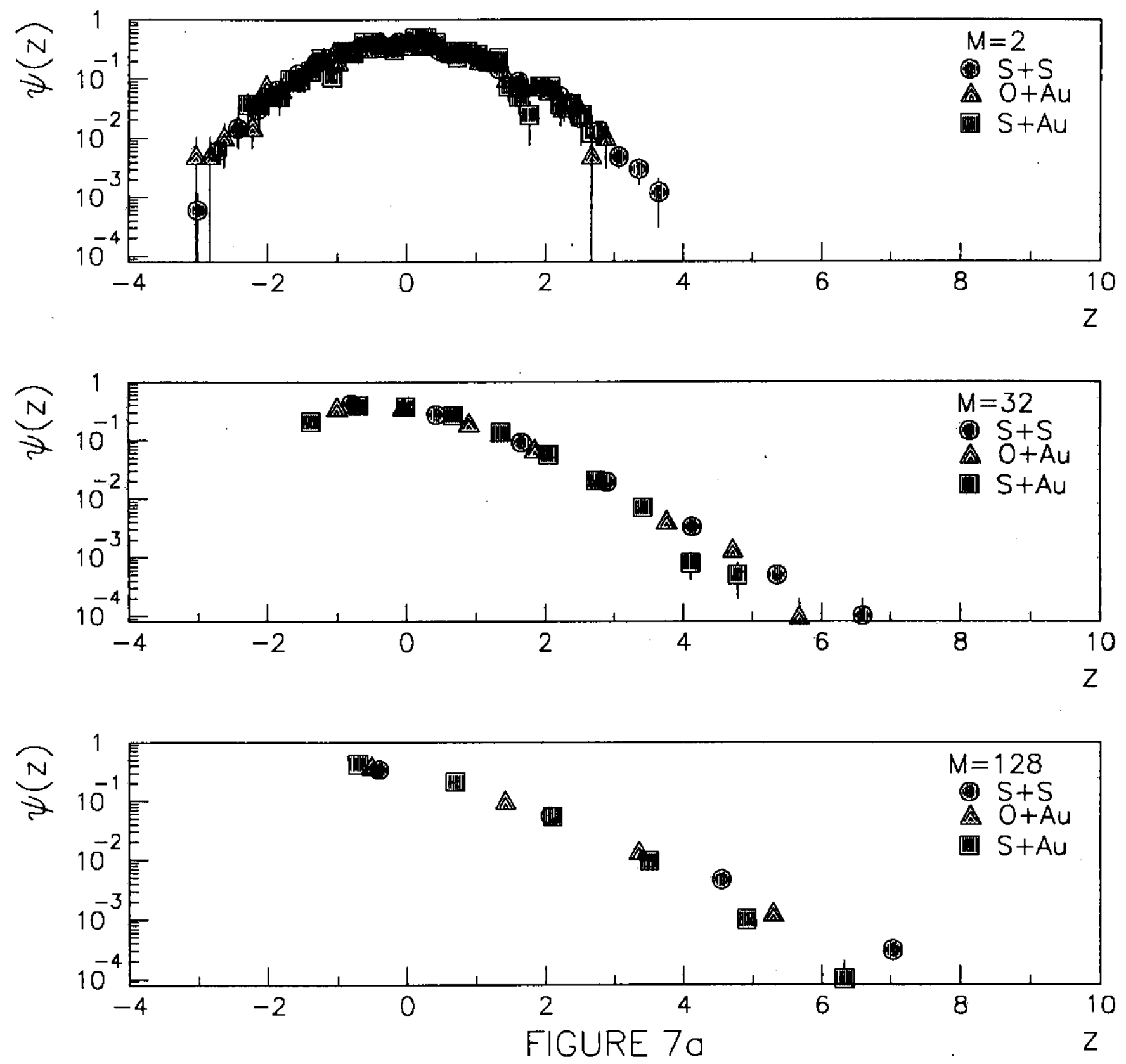

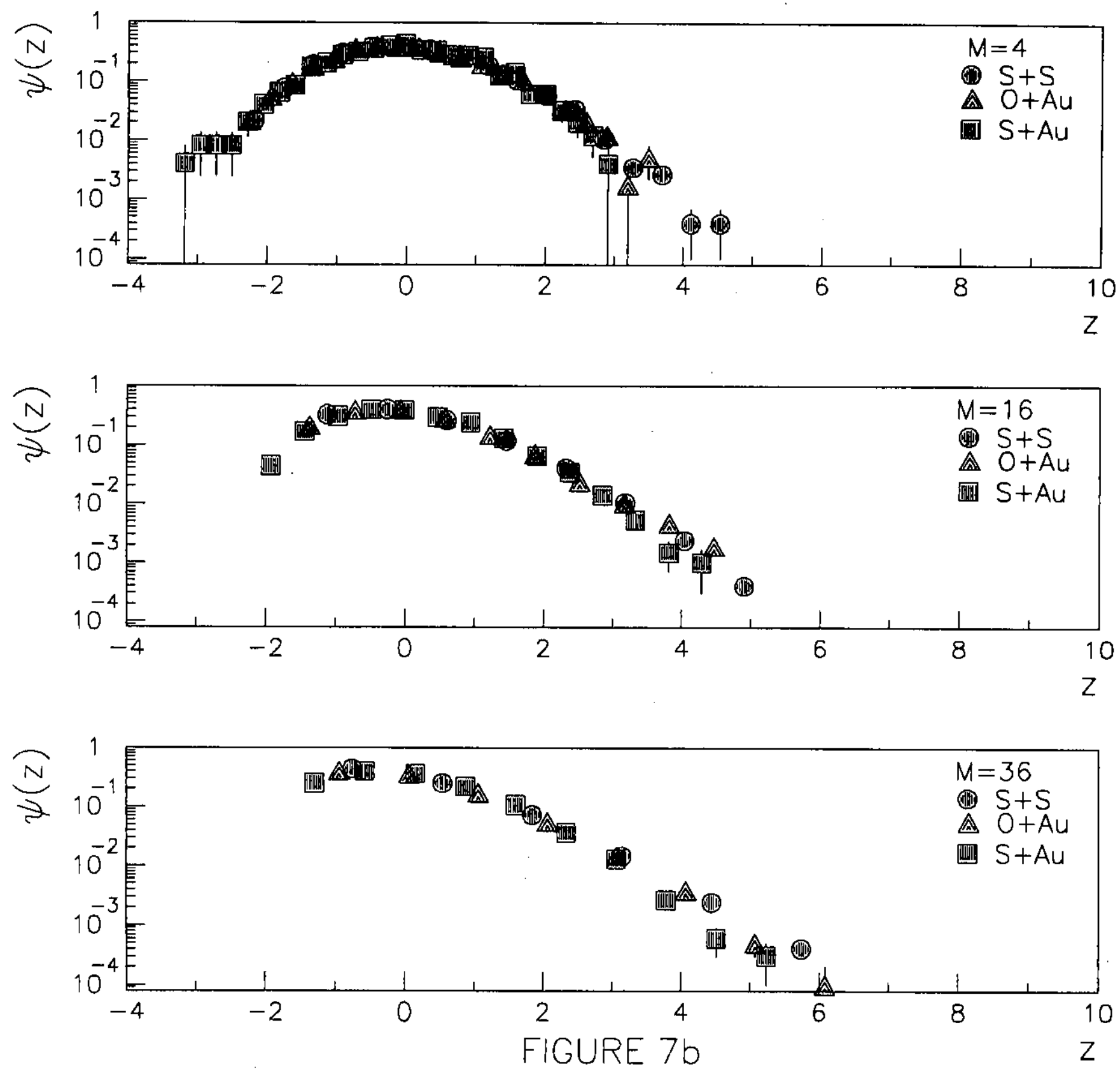

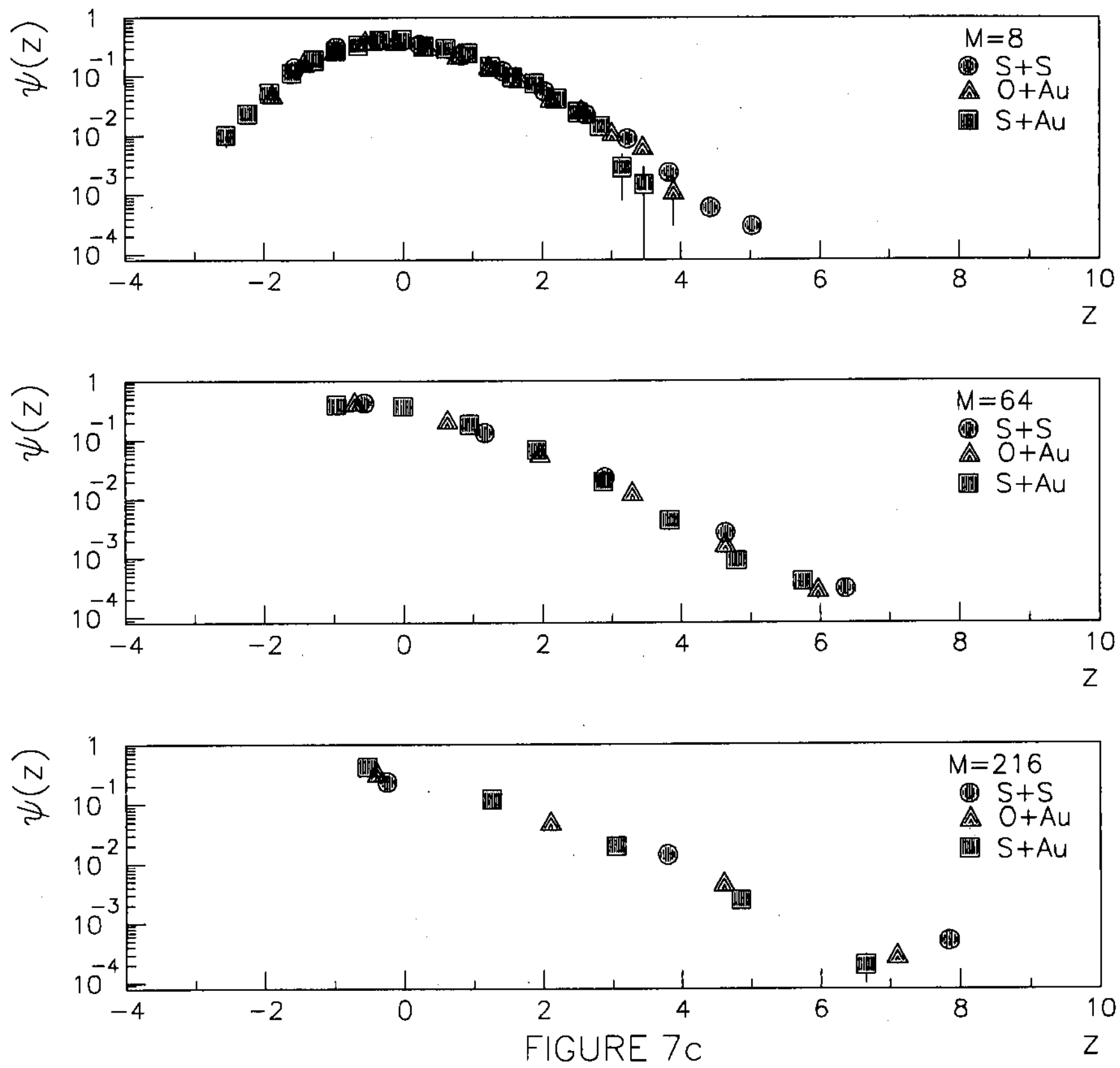

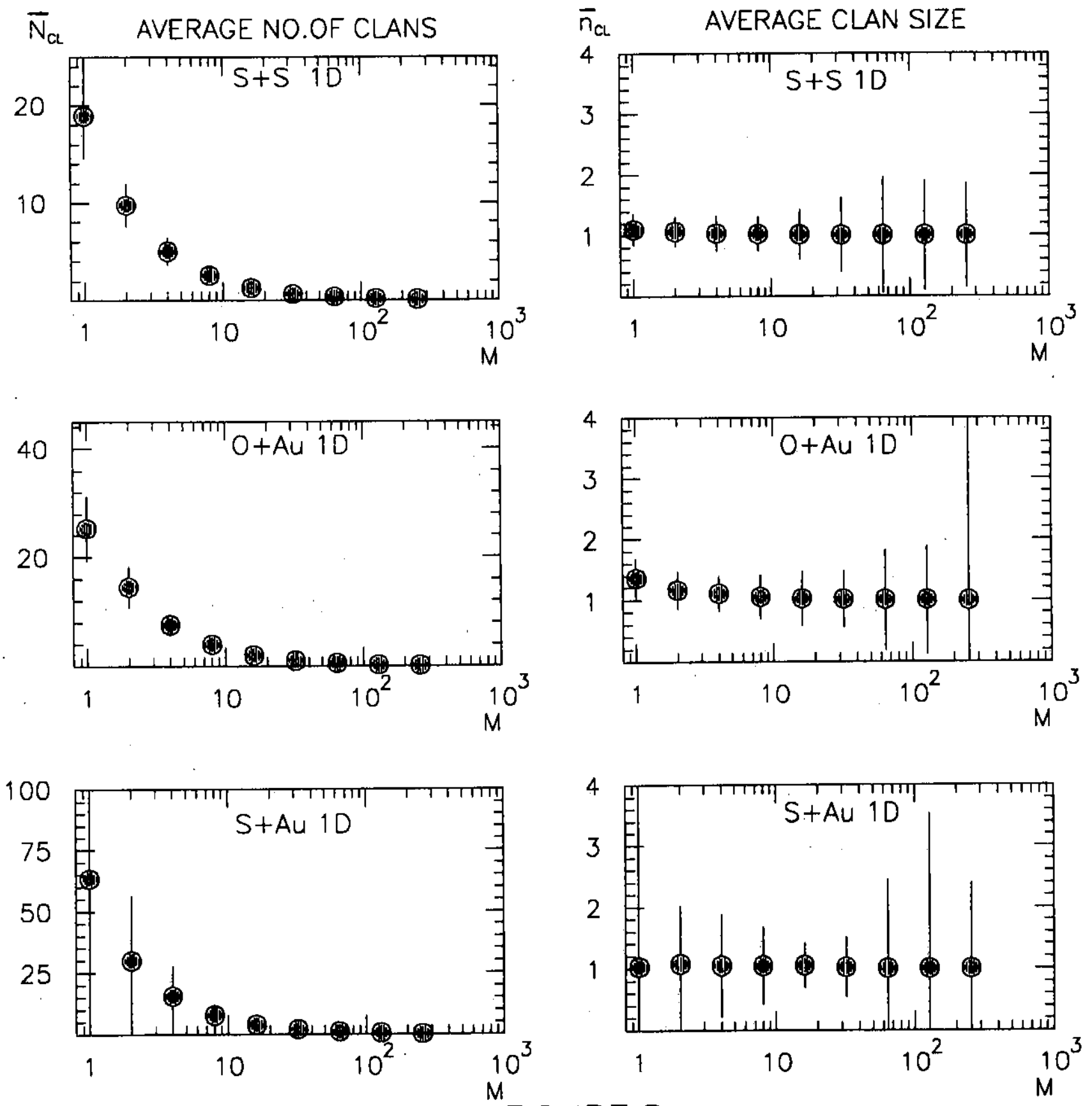

FIGURE 8 a 

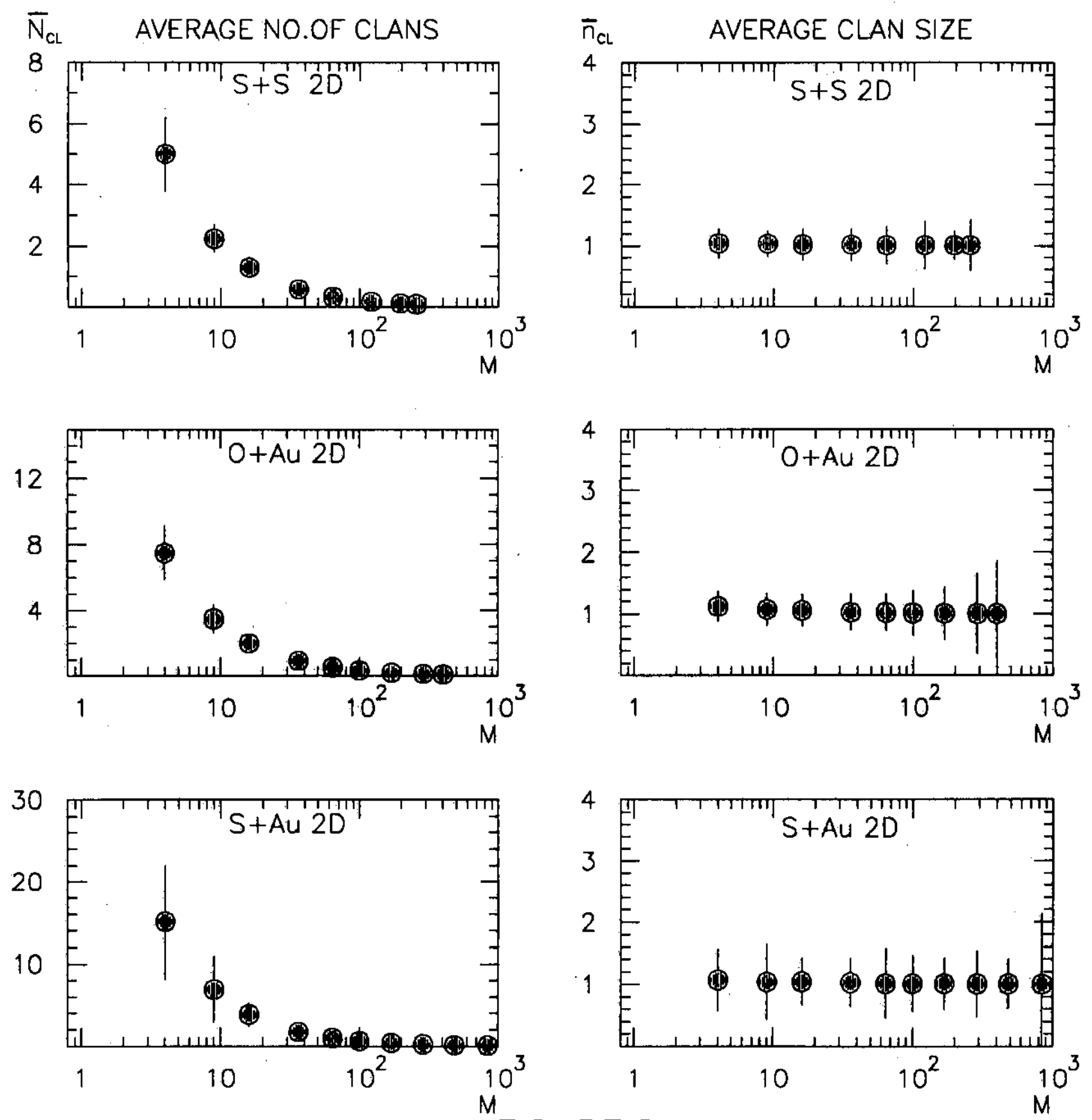

FIGURE 8 b 

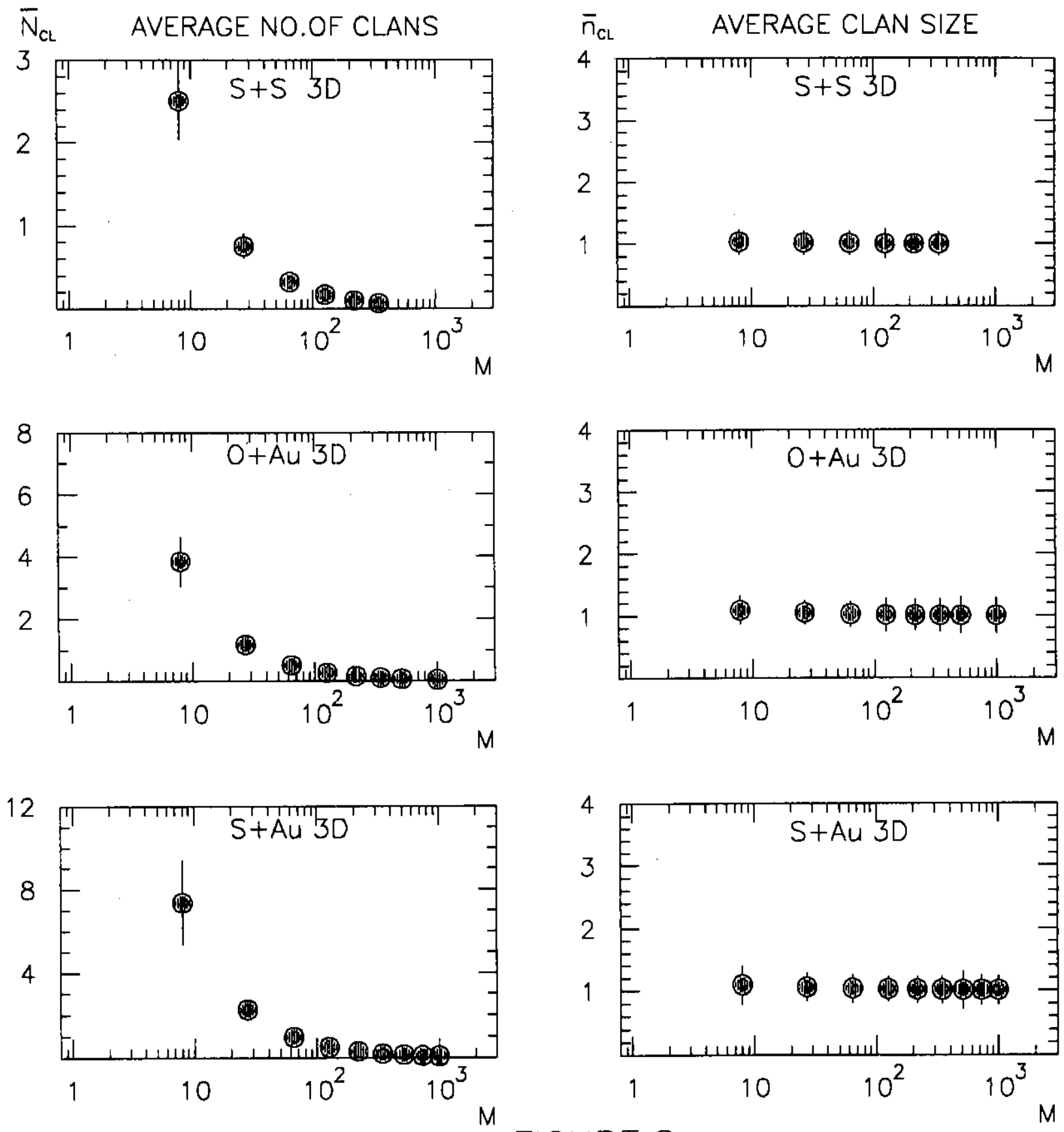

FIGURE $8 \mathrm{c}$ 
$F_{3}$
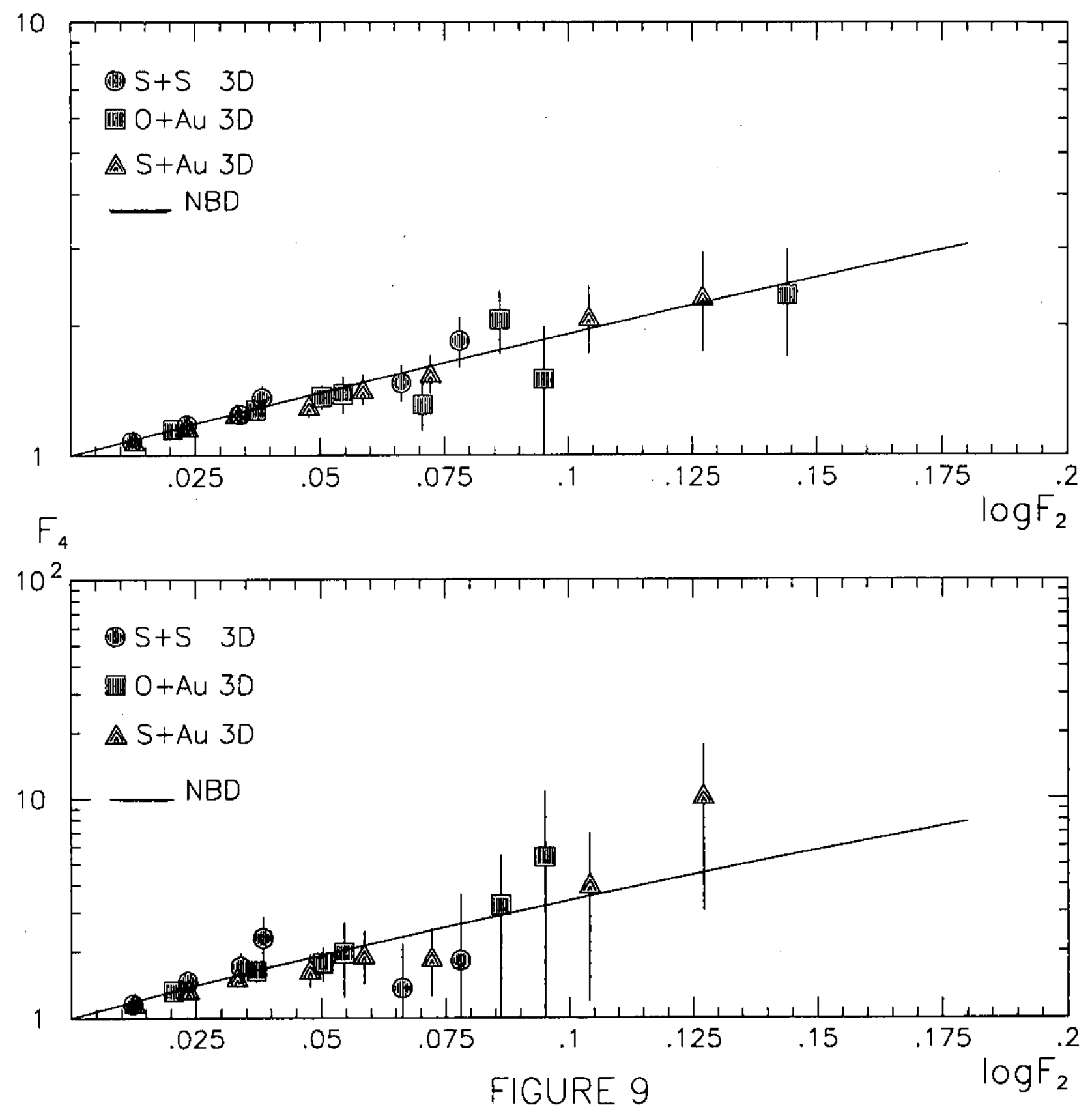